\title{
Novel quantification of the regional strain distribution in the anterior cruciate ligament in response to simulated loading using micro- CT imaging
}

\author{
Alexandra M. Blokker ${ }^{1}$, Ryan Wood ${ }^{2}$, Jaques C. Milner ${ }^{3}$, David W. Holdsworth ${ }^{4}$, Timothy A. Burkhart ${ }^{5^{*}}$ (I) and \\ Alan Getgood ${ }^{6,7}$
}

\begin{abstract}
Purpose: A large percentage of anterior cruciate ligament $(\mathrm{ACL})$ surgical reconstructions experience sub-optimal outcomes within 2 years. A potential factor contributing to poor outcomes is an incomplete understanding of microlevel, regional ACL biomechanics. This research aimed to demonstrate a minimally invasive method that uses micro$\mathrm{CT}$ imaging to quantify regional $\mathrm{ACL}$ strains under clinically relevant joint loading.

Methods: A pattern of $0.8 \mathrm{~mm}$ diameter zirconium dioxide beads were arthroscopically inserted into four regions of the ACL of four cadaveric knee specimens (mean [SD] age $=59$ [9] years). A custom micro-CT compatible joint motion simulator then applied clinically relevant joint loading conditions, while an image was acquired at each condition. From the resulting images, strains within each region were calculated using the centroid coordinates of each tissueembedded bead. Strain repeatability was assessed using the mean intra-specimen standard deviation across repeated load applications. A one-way repeated measures ANOVA $(a=0.05)$ was used to determine regional strain variations.

Results: The mean intra-specimen standard deviation across repeated load application was \pm 0.003 strain for all specimens. No statistically significant differences were found between tissue regions, although medium and large effect sizes (0.095-0.450) suggest that these differences may be clinically relevant.

Conclusions: The method presented here demonstrates a minimally invasive measurement of regional ACL strain under clinically relevant joint loads using micro-CT imaging. The strain measurements demonstrated excellent reliability across the five repeated load applications and suggest a non-homogenous distribution of strain through the ACL.
\end{abstract}

Keywords: Anterior cruciate ligament, Regional strain, Motion simulator, Medical imaging, Cadaveric specimen

\section{Background}

Despite being a highly developed and widely studied procedure, up to $10 \%$ of anterior cruciate ligament reconstruction (ACLR) cases experience acute failure within 2 years post-surgery $[1,20]$. Approximately

\footnotetext{
*Correspondence: timothy.burkhart@utoronto.ca

${ }^{5}$ Kinesiology and Physical Education, University of Toronto, 55 Harbord St, Toronto, ON M5S 2W6, Canada

Full list of author information is available at the end of the article
}

$25 \%$ of patients report persisting instability, which has been highly correlated with altered joint kinematics [20, 23]; subsequently linked to cartilage thinning [2], early onset osteoarthritis [22], reduced joint function, and loss of mobility. One potential factor contributing to sub-optimal surgical outcomes is an incomplete understanding of the micro-level biomechanics during normal physiological use, including regional tissue-strain variations in the native ACL [6]. Thus, in order to reinstate the knee joint's native (i.e., intact) 
kinematics, the native ACL strain distribution should be fully characterized in high resolution under clinically relevant joint loads.

Previous approaches to quantifying intact soft tissue strain in response to joint loading fall into two categories: direct instrumentation of the tissue (e.g., differential variable reluctance transducers [DVRTs]; Hall Effect strain transducers [HESTs]) $[6,17,19]$, and use of radiographic imaging to track tissue-embedded radiopaque markers (e.g., radiostereometric analysis [RSA]). Methods which directly instrument the tissue were inherently restricted to measure strain in only the lower third of the anteromedial bundle (AMB) of the ACL to prevent the surface-mounted instrumentation from impinging with surrounding structures in the joint capsule $[6,19]$. While this provided useful information about the region of tissue directly instrumented, it missed potentially important regional inhomogeneities along the length of the ACL [10]. Past studies have demonstrated potential for using RSA to non-invasively determine the position of radiopaque markers embedded in soft tissue structures as they deform under load $[10,14]$. However, the method has not yet been applied to the measurement of intact tissue strain in response to clinically relevant joint loads. One limitation of this method is RSA imaging resolution. The current gold standard for ligament tissue strain measurement is the DVRT, which can reportedly resolve strain differences as low as 0.0014 strain over a $5 \mathrm{~mm}$ region [3]. Although RSA is a high resolution $(\sim 0.10 \mathrm{~mm})$ imaging modality [4], it cannot capture the same strain resolution as the DVRT. Micro-computed tomography (micro-CT) imaging has demonstrated higher spatial resolutions than RSA, allowing improved visualization of the joint [13, 21]. Thus, micro-CT imaging could provide similar strain measurement resolution as the current gold standard, with the potential to measure multiple regions of the tissue in a minimally-invasive manner. This method has not yet been utilized to quantify regional strain distributions in the ACL.

Therefore, the purpose of this investigation was twofold: i) to demonstrate the feasibility of using micro$\mathrm{CT}$ imaging and radiopaque beads to quantify $\mathrm{ACL}$ strains in an intact cadaveric model in response to clinically relevant (e.g., pivot shift, Lachmans) loading; and ii) to quantify the regional strain distribution along the length of the ACL and to determine if differences exists in different regions in both the axial and trans-axial directions. We hypothesized that there would be regional strain differences through the ACL in response to clinically relevant loading.

\section{Methods}

\section{Specimen preparation}

Four fresh-frozen human cadaveric knee joint specimens (Science Care Inc., AZ, US) (mean [SD] age: 59 [9] years, 1 male, 2 right) with no visible damage to the ACL, posterior cruciate ligament (PCL), or menisci (inspected arthroscopically prior to testing) were used in accordance with tissue use and ethical guidelines (Approval Number: MW 030217). The specimens were kept frozen at $-20^{\circ} \mathrm{C}$ and thawed for approximately $18 \mathrm{~h}$ prior to use. All specimens were sectioned at the mid-femur and mid-tibia and $75 \mathrm{~mm}$ of soft tissue was removed to expose the proximal femur and distal tibia. Soft tissue surrounding the joint was left intact.

With the femur secured to an arthroscopic extremity holder (Model 1650 Sawbones ${ }^{\circledR}$, Pacific Research Laboratories, Inc., WA, US), 14 small diameter $(0.8 \mathrm{~mm}$ diameter) zirconium dioxide $\left(\mathrm{ZrO}_{2}\right)$ beads $(.8 \mathrm{~mm}-\mathrm{c}$ $\mathrm{ZrO}_{2}$ Ball Grade 10, Boca Bearing Inc., FL, US) were arthroscopically implanted into the anteromedial bundle (AMB) of the ACL by a fellowship-trained orthopedic surgeon. Beads were embedded into the soft tissue with an 18-gauge needle (BD Precision Glide, NJ, US) and the depth was controlled using the top of the needle bevel as a reference $(\sim 3 \mathrm{~mm})$. The beads were embedded approximately $3-5 \mathrm{~mm}$ apart in two axially directed (i.e., along the length of the ACL) columns spaced approximately 3-5 $\mathrm{mm}$ apart in the trans-axial (i.e., along the width of the ACL) direction in the following regions (Fig. 1): i) four beads were embedded in the femoral footprint in a square configuration with two directly at the ligament enthesis; ii) six beads were embedded in three pairs at the mid-substance; and iii) four beads were embedded in the tibial footprint, with two at the enthesis. Once the beads were embedded, the exposed tibia and femur bone segments were potted into $75 \mathrm{~mm}$ and $50 \mathrm{~mm}$ diameter sections of ABS tubing, respectively, via dental cement (Denstone Dental Cement, Hereaus Holdings GmbH, Hanau, Germany) [7]. Previous research has shown that insertion of the beads does not affect the mechanical properties of the tissues and the beads do not move within the tissue [8].

\section{Experimental protocol}

The potted specimens were rigidly secured within a custom-designed and validated micro-CT compatible five degree-of-freedom (DOF) knee joint motion simulator [7]. Briefly, the simulator applies closed-loop load control to the cadaveric knee while the joint is centered within the bore of a micro-CT scanner (GE Locus Ultra, London, Ontario; standard anatomical protocol: $80 \mathrm{kVp}$, $50 \mathrm{~mA}, 0.15 \mathrm{~mm}$ isotropic voxels; $16 \mathrm{~s}$ scan time); all loads 


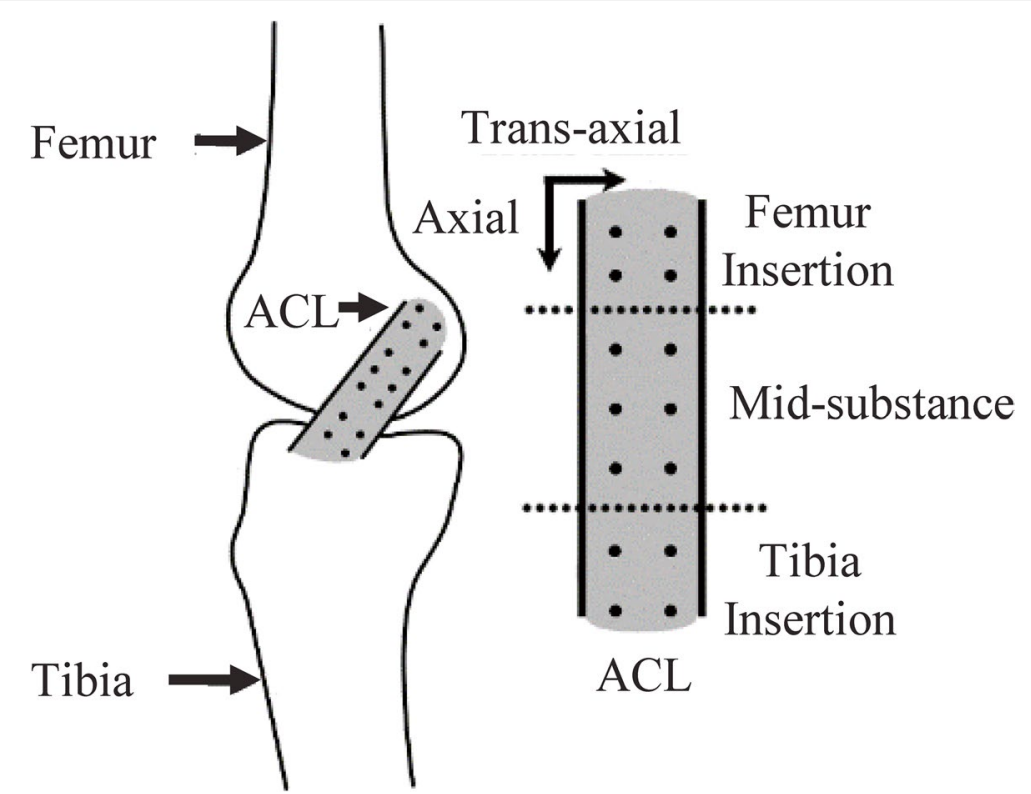

Fig. 1 Schematic representation of the knee joint and ACL showing the approximate arrangement of beads in the ACL sub-divided into the femur insertion, mid-substance, and tibia insertion regions

were applied to the tibia while the femur remained fixed. The specimens were pre-conditioned with ten cycles of joint distraction at $0.25 \mathrm{~Hz}$ from -90 to $90 \mathrm{~N}$, ten cycles of internal rotation from 0 to $5 \mathrm{Nm}$ at $0.25 \mathrm{~Hz}$, followed by ten cycles of anterior load from 0 to $100 \mathrm{~N}$ at $0.25 \mathrm{~Hz}$. A baseline (i.e., unloaded) image of the joint was acquired with a $10 \mathrm{~N}$ compressive load for joint stabilization, followed by a series of loading conditions: i) $5 \mathrm{Nm}$ internal rotation with a $10 \mathrm{~N}$ compressive load; ii) $100 \mathrm{~N}$ anterior translation force with a $10 \mathrm{~N}$ compressive load; and iii) a simulated pivot shift composed of a $100 \mathrm{~N}$ compression force, $5 \mathrm{Nm}$ internal rotation, $10 \mathrm{Nm}$ valgus rotation, and $100 \mathrm{~N}$ anterior translation force. Each of load condition was repeated five times at $0^{\circ}, 15^{\circ}$, and $30^{\circ}$ of knee flexion with an image acquired at each the load targets. A new baseline image was acquired at each new flexion angle. The scan was initiated immediately upon reaching the target force or moment and takes approximately $16 \mathrm{~s}$ to complete. Previous research evaluating this system suggests there is no noticeable creep in the system over this duration [7].

\section{Data analysis}

The centroid location ( $x, y, z$ coordinate) of each bead was computed using custom developed software using a user input threshold (in HU) and user input seed points to create a cube around the surface of each bead. The centroid of the cube was then calculated in the scanner coordinate system. After the beads were located, a second, custom designed software program (MATLAB
R2017b, MathWorks, MA, US; standard toolkits) was used to calculate the tissue strains. This program calculated a change in length within the ligament by comparing the Euclidean distance between adjacent beads before and after an applied load. These two inter-bead distances were then used to calculate tissue strain.

The bead pattern allowed strain to be calculated within the following regions of the ACL: i) the femoral footprint; ii) the superior mid-substance region (mid 1); iii) the inferior mid-substance region (mid 2); iv) the tibia footprint; and v) a global axial strain calculated between beads embedded in the femur and tibia footprints. The double column configuration allowed for trans-axial strains to be measured in each of these regions. A previous study that evaluated this strain calculation method found that it is accurate and precise, measuring to within 0.006 RMSE for strains as low as 0.007 strain using the same scanning and threshold parameters [9].

\section{Statistical analysis}

Across-trial reliability was calculated using a oneway repeated measures ANOVA (with trial number as the independent variable) and intraclass correlation coefficients (ICCs) (two-way random, absolute agreement, single measures). The following ICC intervals were used to define the magnitude of reliability [11]: ICC $<0.4=$ poor, $0.4<\mathrm{ICC}<0.59=$ fair, $0.6<\mathrm{ICC}<0.74=$ good, and $\mathrm{ICC}>0.74=$ excellent. Variability that can be attributed to the measurement technique was assessed by calculating the mean 
intra-specimen standard deviation across repeated load applications for each specimen. This was repeated for all load conditions. A set of one-way repeated measures ANOVAs were then performed to determine if the strain varied significantly across the different regions of the ACL. The ANOVAs were performed independently for the axial and trans-axial strains, and post-hoc comparisons were conducted using a Bonferroni adjustment. Effect sizes were also calculated in the form of the partial eta squared $\left(\eta^{2}\right)$ and evaluated according to Maher et al. [18]. All statistics were performed using SPSS (Version 25, IBM, NY, US) with $\alpha=0.05$ for all tests.

\section{Results}

Bead placement

All images provided sufficient contrast to threshold the radiopaque markers $\left(\mathrm{ZrO}_{2}>32,767 \mathrm{HU}\right)$ from surrounding tissues (e.g., cortical bone $=2000-3000 \mathrm{HU}$, soft tissue $=100-300 \mathrm{HU})$. The entire joint capsule was captured within the imaging FOV for all loads and flexion angles and the different regions were distinguishable (Fig. 2).

\section{Strain variability}

One specimen was not tested in anterior translation or pivot shift loads at $30^{\circ}$ of flexion due to interference with the imaging bore; therefore these cases were reported for the three specimens completed. No significant differences
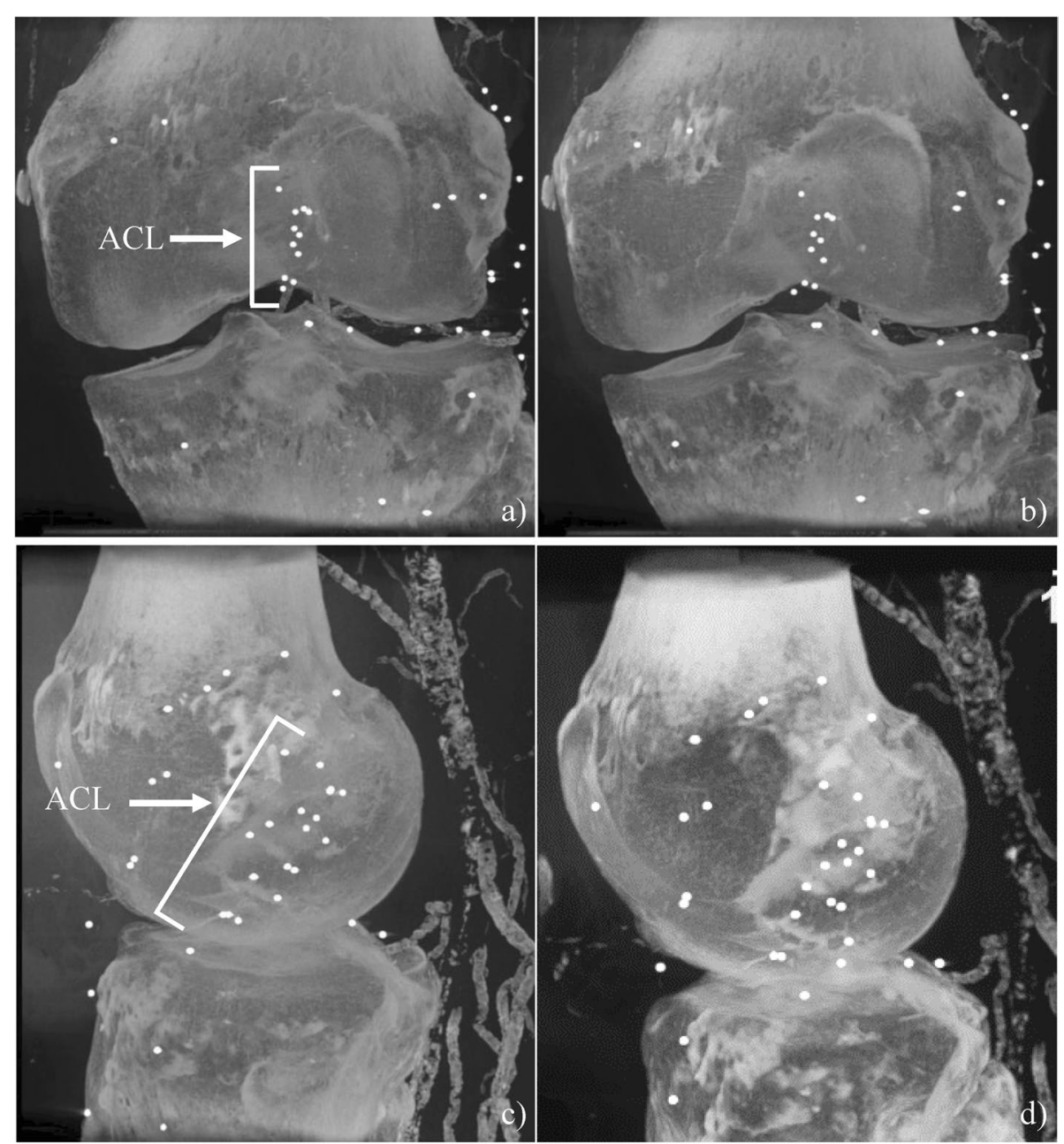

Fig. 2 Maximum Intensity Projections (MIP) of a cadaveric specimen before [a coronal view, c sagittal view], and after [b coronal view, d sagittal view] a simulated pivot shift with loads of $100 \mathrm{~N}$ compression, $5 \mathrm{Nm}$ internal rotation, $10 \mathrm{Nm}$ valgus rotation, and $100 \mathrm{~N}$ anterior translation force at $0^{\circ}$ flexion. The patella was digitally removed in post-processing in these images, for ease of viewing the joint capsule. Additional beads were also implanted into the lateral soft tissue structures but were analyzed as part of a subsequent study 
were found in the strain measured in each region across the five repeated trials for the axial strains $(p>0.05)$ with the exception of three cases: i) femur region at $30^{\circ}$ flexion, $5 \mathrm{Nm}$ IR $(p=0.045)$, ii) inferior mid-substance region at $30^{\circ}$ flexion, pivot shift $(p=0.025)$, and iii) global strain at $30^{\circ}$ flexion, $5 \mathrm{Nm} \mathrm{IR}(p=0.032)$ (Table 1$)$. Post hoc tests of these cases failed to locate where the significant differences occurred. Repeated axial strain measurements were found to have excellent reliability for all cases with ICCs greater than 0.799 (Table 1).

No significant differences were found across repeated trials for the trans-axial strains with the exception of four regions: i) inferior mid-substance region at $0^{\circ}$ flexion angle, $5 \mathrm{Nm}$ IR $(p=0.006)$, ii) inferior mid-substance under $15^{\circ}$ flexion angle, pivot shift $(p=0.016)$, iii) the tibia region at $30^{\circ}$ flexion angle, $5 \mathrm{Nm}$ IR $(p=0.037)$, and iv) inferior mid-substance region under $30^{\circ}$ flexion angle, $100 \mathrm{~N}$ AT $(p=0.009)$ (Table 2$)$. However, posthoc tests failed to find significance for any of these cases. Repeated trans-axial strain measurements showed excellent reliability in $96 \%$ of cases, fair reliability in $2 \%$ of the cases (one case: $15^{\circ}$ flexion angle, $5 \mathrm{Nm} \mathrm{IR}$, in the femur region), and a negative ICC value in the remaining $2 \%$ of cases (one case: $30^{\circ}$ flexion angle, $100 \mathrm{~N}$ AT in the femur region) (ICC $=-0.05)$ (Table 2).

The mean intra-specimen standard deviation across repeated load application was 0.003 strain in the axial direction for all specimens, across all load conditions

Table 1 ICC and $p$ values calculated for axial strains measured across five repeated trials at each load condition, for each region in the AMB

\begin{tabular}{|c|c|c|c|c|c|c|c|c|c|c|c|}
\hline \multirow[t]{2}{*}{$\begin{array}{l}\text { Flexion } \\
\text { Angle }\left({ }^{\circ}\right)\end{array}$} & \multirow[t]{2}{*}{ Load Condition } & \multicolumn{2}{|c|}{ Femur Insertion } & \multicolumn{2}{|c|}{$\begin{array}{l}\text { Superior Mid- } \\
\text { Substance }\end{array}$} & \multicolumn{2}{|c|}{$\begin{array}{l}\text { Inferior Mid- } \\
\text { Substance }\end{array}$} & \multicolumn{2}{|c|}{ Tibia Insertion } & \multicolumn{2}{|l|}{ Global } \\
\hline & & ICC & $p$ value & ICC & $p$ value & ICC & $p$ value & ICC & $p$ value & ICC & $p$ value \\
\hline \multirow[t]{3}{*}{0} & $5 \mathrm{Nm} I \mathrm{R}$ & 0.971 & 0.233 & 0.969 & 0.488 & 0.999 & 0.924 & 0.951 & 0.411 & 0.998 & 0.968 \\
\hline & $100 \mathrm{~N} A \mathrm{~T}$ & 0.934 & 0.488 & 0.984 & 0.661 & 0.994 & 0.762 & 0.948 & 0.297 & 0.973 & 0.738 \\
\hline & Pivot Shift & 0.892 & 0.243 & 0.969 & 0.202 & 0.998 & 0.148 & 0.962 & 0.359 & 0.962 & 0.359 \\
\hline \multirow[t]{3}{*}{15} & $5 \mathrm{Nm} \mathrm{IR}$ & 0.987 & 0.966 & 0.966 & 0.459 & 0.986 & 0.574 & 0.82 & 0.63 & 0.920 & 0.448 \\
\hline & $100 \mathrm{~N} A T$ & 0.999 & 0.115 & 0.993 & 0.061 & 0.999 & 0.888 & 0.835 & 0.394 & 1.000 & 0.799 \\
\hline & Pivot Shift & 0.999 & 0.71 & 0.99 & 0.685 & 0.998 & 0.148 & 0.985 & 0.438 & 1.000 & 0.124 \\
\hline \multirow[t]{3}{*}{30} & $5 \mathrm{Nm} \mathrm{IR}$ & 0.99 & $0.045^{*}$ & 0.964 & 0.171 & 0.994 & 0.902 & 0.919 & 0.689 & 0.997 & $0.032^{*}$ \\
\hline & $100 \mathrm{~N} A \mathrm{~T}$ & $0.991^{\mathrm{a}}$ & 0.434 & $0.799^{a}$ & 0.112 & $0.999^{\mathrm{a}}$ & 0.981 & $0.94^{\mathrm{a}}$ & 0.419 & $0.993^{\mathrm{a}}$ & 0.216 \\
\hline & Pivot Shift & $0.915^{\mathrm{a}}$ & 0.981 & $0.964^{\mathrm{a}}$ & 0.172 & $0.96^{\mathrm{a}}$ & $0.025^{*}$ & $0.974^{\mathrm{a}}$ & 0.555 & $0.999^{a}$ & 0.106 \\
\hline
\end{tabular}

IR Internal Rotation, AT Anterior Translation Force

* Post-hoc analysis failed to find significant difference

a case with $n=3$

Table 2 ICC and p values calculated for trans-axial strains measured across five repeated trials at each load condition, for each region in the $\mathrm{ACL}$

\begin{tabular}{|c|c|c|c|c|c|c|c|c|c|}
\hline \multirow{2}{*}{$\begin{array}{l}\text { Flexion } \\
\text { Angle }\left({ }^{\circ}\right)\end{array}$} & \multirow[t]{2}{*}{ Load Condition } & \multicolumn{2}{|c|}{ Femur Insertion } & \multicolumn{2}{|c|}{ Superior Mid-Substance } & \multicolumn{2}{|c|}{ Inferior Mid-Substance } & \multicolumn{2}{|c|}{ Tibia Insertion } \\
\hline & & ICC & $p$ value & ICC & $p$ value & ICC & $p$ value & ICC & $p$ value \\
\hline \multirow[t]{3}{*}{0} & $5 \mathrm{Nm} I \mathrm{R}$ & 0.996 & 0.711 & 0.998 & $0.006^{*}$ & 0.988 & 0.553 & 0.969 & 0.599 \\
\hline & $100 \mathrm{~N}$ AT & 0.984 & 0.174 & 0.964 & 0.905 & 0.964 & 0.629 & 0.995 & 0.466 \\
\hline & Pivot Shift & 0.955 & 0.234 & 0.993 & 0.344 & 0.999 & 0.800 & 0.993 & 0.089 \\
\hline \multirow[t]{3}{*}{15} & $5 \mathrm{Nm} \mathrm{IR}$ & 0.524 & 0.438 & 0.985 & 0.952 & 0.991 & 0.985 & 0.994 & 0.953 \\
\hline & $100 \mathrm{~N} A T$ & 0.999 & 0.921 & 0.991 & 0.055 & 0.999 & 0.832 & 0.984 & 0.978 \\
\hline & Pivot Shift & 0.999 & 0.541 & 0.989 & 0.617 & 0.999 & 0.016 & 0.984 & 0.978 \\
\hline \multirow[t]{3}{*}{30} & $5 \mathrm{Nm} I \mathrm{R}$ & 0.993 & 0.445 & 0.999 & 0.964 & 0.988 & 0.882 & 0.981 & $0.037^{*}$ \\
\hline & $100 \mathrm{~N}$ AT & $-0.05^{\mathrm{a}}$ & 0.583 & $0.999^{a}$ & 0.417 & $0.996^{\mathrm{a}}$ & $0.009^{*}$ & $0.966^{\mathrm{a}}$ & 0.951 \\
\hline & Pivot Shift & $0.984^{\mathrm{a}}$ & 0.694 & $0.971^{\mathrm{a}}$ & 0.383 & $0.987^{a}$ & 0.183 & $0.998^{\mathrm{a}}$ & 0.302 \\
\hline
\end{tabular}

IR Internal Rotation, AT Anterior Translation Force

* Post-hoc analysis failed to find significant difference

a Case with $n=3$ 
(Table 3). The largest variability in the strains occurred at the femur insertion and superior mid-substance regions (mean $\mathrm{SD}=0.004$ strain), with the lowest variability in the tibia insertion region (mean $\mathrm{SD}=0.002$ strain). The intra-specimen variability was relatively consistent for each specimen (Table 3 ).

The mean intra-specimen standard deviation across repeated load application was 0.006 strain in the trans-axial direction for all specimens, across all load conditions. The largest variability in the trans-axial strains occurred at the superior mid-substance (mean $\mathrm{SD}=0.008$ strain), with the lowest variability in the femur insertion region (mean $\mathrm{SD}=0.005$ strain). The intra-specimen variability was relatively consistent for each specimen (Table 3 ).

\section{Regional variation in axial strain}

No statistically significant differences were found between regional axial strains when the knee was exposed to a $5 \mathrm{Nm}$ internal rotation load $(p>0.05)$ (Fig. 3a), a $100 \mathrm{~N}$ anterior translation load $(p>0.05)$ (Fig. 3b), or a simulated pivot shift $(p>0.05)$ (Fig. 3c) at any of the flexion angles tested. However, medium and large effect sizes were found for all regions and degrees of flexion $\left(\eta^{2}=0.107-0.448\right)$ (Table 4$)$.

\section{Regional variation in trans-axial strain}

Similarly, no statistically significant regional differences were found between regional trans-axial strains when the knee was exposed to a $5 \mathrm{Nm}$ internal rotation load $(p>0.05)$ (Fig. 4a), a $100 \mathrm{~N}$ anterior translation load $(p>0.05)$ (Fig. $4 \mathrm{~b})$, or the simulated pivot shift $(p>0.05)$ (Fig. 4c) for any flexion angle tested. However, similar

Table 3 The mean of the standard deviations for the axial and trans-axial strains measured across repeated trials for each region of the ligament, for each specimen. This represents a mean of all load conditions tested for each specimen

\begin{tabular}{cllll}
\hline Specimen & $\begin{array}{l}\text { Femur } \\
\text { Insertion } \\
\text { (strain) }\end{array}$ & $\begin{array}{l}\text { Superior Mid- } \\
\text { Substance } \\
\text { (strain) }\end{array}$ & $\begin{array}{l}\text { Inferior Mid- } \\
\text { Substance } \\
\text { (strain) }\end{array}$ & $\begin{array}{l}\text { Tibia } \\
\text { Insertion } \\
\text { (strain) }\end{array}$ \\
\hline Axial & & & & \\
1 & 0.001 & 0.002 & 0.003 & 0.004 \\
2 & 0.009 & 0.007 & 0.003 & 0.001 \\
3 & 0.002 & 0.003 & 0.004 & 0.002 \\
4 & 0.005 & 0.005 & 0.002 & 0.001 \\
Trans-axial & & & & 0.004 \\
1 & 0.002 & 0.010 & 0.003 & 0.008 \\
2 & 0.009 & 0.013 & 0.010 & 0.002 \\
3 & 0.004 & 0.009 & 0.017 & 0.009 \\
4 & 0.002 & 0.002 & 0.004 & \\
\hline
\end{tabular}

to the axial strains, medium and large effect sizes were found for all loading conditions and at all flexion angles $\left(\eta^{2}=0.095-0.376\right)($ Table 4$)$.

\section{Discussion}

To the author's knowledge this is the first study to quantify regional ACL strain in response to clinically relevant joint loads (e.g., pivot shift and Lachmans) using a minimally invasive micro-CT imaging-based method. The strains measured under various joint loading conditions demonstrated excellent reliability across repeated load applications, indicating that the method is highly consistent in both strain measurement and load application. No statistically significant differences were found across the five regions of the AMB in either the axial or trans-axial directions; however, effect sizes suggest potential clinically meaningful regional strain variability.

The minimally-invasive strain measurement method described here was highly repeatable, as indicated by low standard deviations in strain measured across the repeated load application trials. It was found that within a set of repeated load applications, the measured interbead strains varied on average by only 0.003 strain between trials. The method's high repeatability is further supported by a lack of statistically significant differences and high ICC values in the strains measured under repeated load application. The totality of this data indicates that the majority of the variation in strain is likely attributable to inherent biological variability (across and within specimens) as opposed to the measurement technique.

The selection of a micro-CT imaging system allows the imaging of both the bone and soft tissue structures within the joint capsule envelopment of an intact tissue specimen. This is advantageous as it ensures the simultaneous measurement of both tissue strain and joint kinematics in high resolution, thus eliminating the need to register independent kinematic tracking and strain measurement systems. The error associated with $2 \mathrm{D}$ to $3 \mathrm{D}$ registration has been shown to be approximately $1.31 \mathrm{~mm}$ for in-plane and $2.40 \mathrm{~mm}$ for out-of-plane motion [15], while fiducial and surface based registration methods can produce errors on the order of 1.25 and $0.25 \mathrm{~mm}$ respectively [16]. These errors are essentially eliminated with the method presented here.

The current investigation demonstrated that this method is capable of locating small diameter $\mathrm{ZrO}_{2}$ beads embedded in soft tissue to quantify regional tissue strains. The resulting images provided a clear distinction between the bone tissue and beads without motion or material artifacts. No statistically significant differences in the distribution of strain were found throughout the AMB in either the axial or trans-axial directions. 

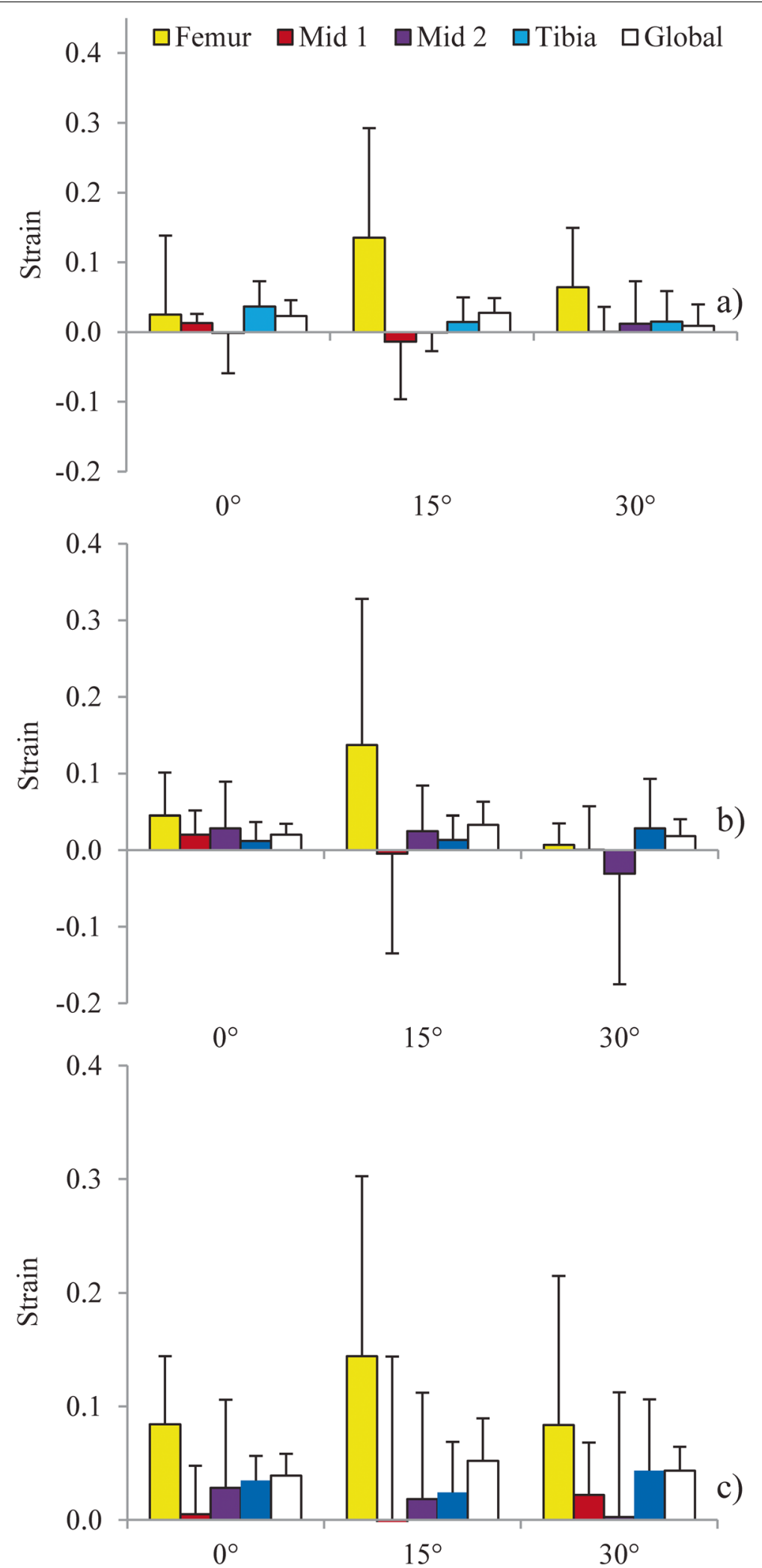

Fig. 3 Comparison of the mean (SD) magnitude of axial strain between ACL regions and across knee flexion angles when exposed to: $\mathbf{a} 5 \mathrm{Nm}$ internal rotation; b $100 \mathrm{~N}$ anterior translation; and $\mathbf{c}$ simulated pivot shift 
Table 4 Effect sizes for each of the loading conditions across the three knee flexion angles tested

\begin{tabular}{llll}
\hline & \multicolumn{2}{l}{ Flexion Angle } & \\
\cline { 2 - 4 } Loading Condition & $\mathbf{0}^{\circ}$ & $\mathbf{1 5}^{\circ}$ & $\mathbf{3 0 ^ { \circ }}$ \\
\hline Axial & & & \\
5 Nm IR & 0.188 & 0.566 & 0.416 \\
100 N AT & 0.166 & 0.104 & 0.255 \\
Pivot Shift & 0.326 & 0.200 & 0.451 \\
Trans-axial & & & \\
5 Nm IR & 0.145 & 0.631 & 0.376 \\
100 N AT & 0.130 & 0.095 & 0.127 \\
Pivot Shift & 0.139 & 0.153 & 0.250 \\
\hline
\end{tabular}

However, medium to large effect sizes found for all conditions suggest that regional strain variation may be clinical relevant and require further investigation. Butler et al. [10] also demonstrated no statistically significant variation in strain distribution along the long axis of the $\mathrm{ACL}$ when a cadaveric human AMB was dissected into five separate fiber bundles while maintaining the femur and tibia footprints. Butler et al. [10] showed a symmetric strain distribution along the longitudinal axis (i.e., from femur insertion to tibia insertion) of each subunit, with the two footprints demonstrating greater, non-significant, strain compared to the mid-substance $(0.0025$ vs. 0.01 strain, respectively). In the present study, a similar trend of higher strains in the footprints was observed when the joint was at $15^{\circ}$ of flexion under a $5 \mathrm{Nm}$ internal rotation load with non-significant difference between the footprints and mid-substance (approximately 0.02 to 0.07 strain). A similar observation was made by Hirokawa et al. [12], who proposed that increased tension at the footprints was a symptom of the un-natural insertion configuration experienced when the ligament undergoes uni-axial tension as opposed to a true in-homogeneity; however, this study was conducted with sub-units from only a single cadaveric specimen. This differs drastically from the approach presented in the present study, where the native ACL and surrounding joint structures were kept fully intact and the ligament may have been strained in more clinically and physiologically accurate directions through actuation of the femur and tibia.

The magnitude of strain measured from the methodology presented here agrees with previously reported values [5]. Berns et al. [5], instrumented cadaveric AMBs with liquid-mercury strain gauges and applied a range of passive joint motions and found, for example, a mean (SD) strain of $0.037(0.018)$ under a pure $100 \mathrm{~N}$ anterior load at a $30^{\circ}$ flexion; this agrees with the current method (0.035 [0.010)] strain) under the same load and flexion angle. Berns et al. [5] also demonstrated a mean (SD) strain of 0.049 (0.014) under a combined load of $100 \mathrm{~N}$ anterior load and $20 \mathrm{Nm}$ of valgus torque, which is similar to the current study's finding of $0.043(0.010)$ in response to the simulated pivot shift. However, different strain responses were found under a $5 \mathrm{Nm}$ internal rotation load at $30^{\circ}$ flexion between the two methods, with Berns et al. [5] finding a mean (SD) strain of 0.014 $(0.012)$ and the present study finding a mean (SD) strain of $-0.008(0.027)$. One possible explanation for this difference is that Berns et al. [5] used a high stiffness sheath around the strain gauge to prevent impingement of the gauge against surrounding structures in the joint and observed that the sheath itself may have impinged with the joint during this motion; highlighting the benefit of a minimally invasive strain measurement method. Furthermore, the variability in the strain measurements between specimens found with the current method was comparable to the variability found by Berns et al. [5], indicating that despite the small sample size in the present study ( $n=4$ vs $n=13$ by Berns et al. [5]). The strains measured can be considered relatively representative of a larger sample.

An interesting finding from this work was a compressive strain demonstrated at a $30^{\circ}$ flexion angle when the joint was subjected to pure internal rotation and anterior loads. However, while the directionality of these strains (negative) is traditionally interpreted as compression, it is more likely, given the type and location of the tissue being tested, that this is indicative of the tissue folding onto itself and not that it is being compressed. A recent study by Hirokawa et al. [12] analyzing strain inhomogeneities using a photoelastic coating to detect fringes in the tissue found regional compressive strain at the tibial insertion with a corresponding tension in the femoral insertion at low joint flexion angles [12]. It was suggested that this was a result of the ligament having no compressive strength and therefore collapsing or folding over itself when the ligament is unloaded, which occurs at specific flexion angles [12]. In the study presented here, tissue relaxation appeared to occur through multiple regions in the AMB, including the superior mid-substance, tibia insertion, and global strain measurements.

There are some limitations that exist with the present study. First, this study was performed on a small sample of cadaveric specimens, and likely contributed to the statistical tests not finding significant differences between the regions. Second, only the AMB was instrumented as result of the small working space afforded by the arthroscopic bead insertion technique. Another limitation of this study was the difficulty of reproducibly placing the radiopaque markers in the same anatomical positions in different specimens. The surgeon implanting the markers 

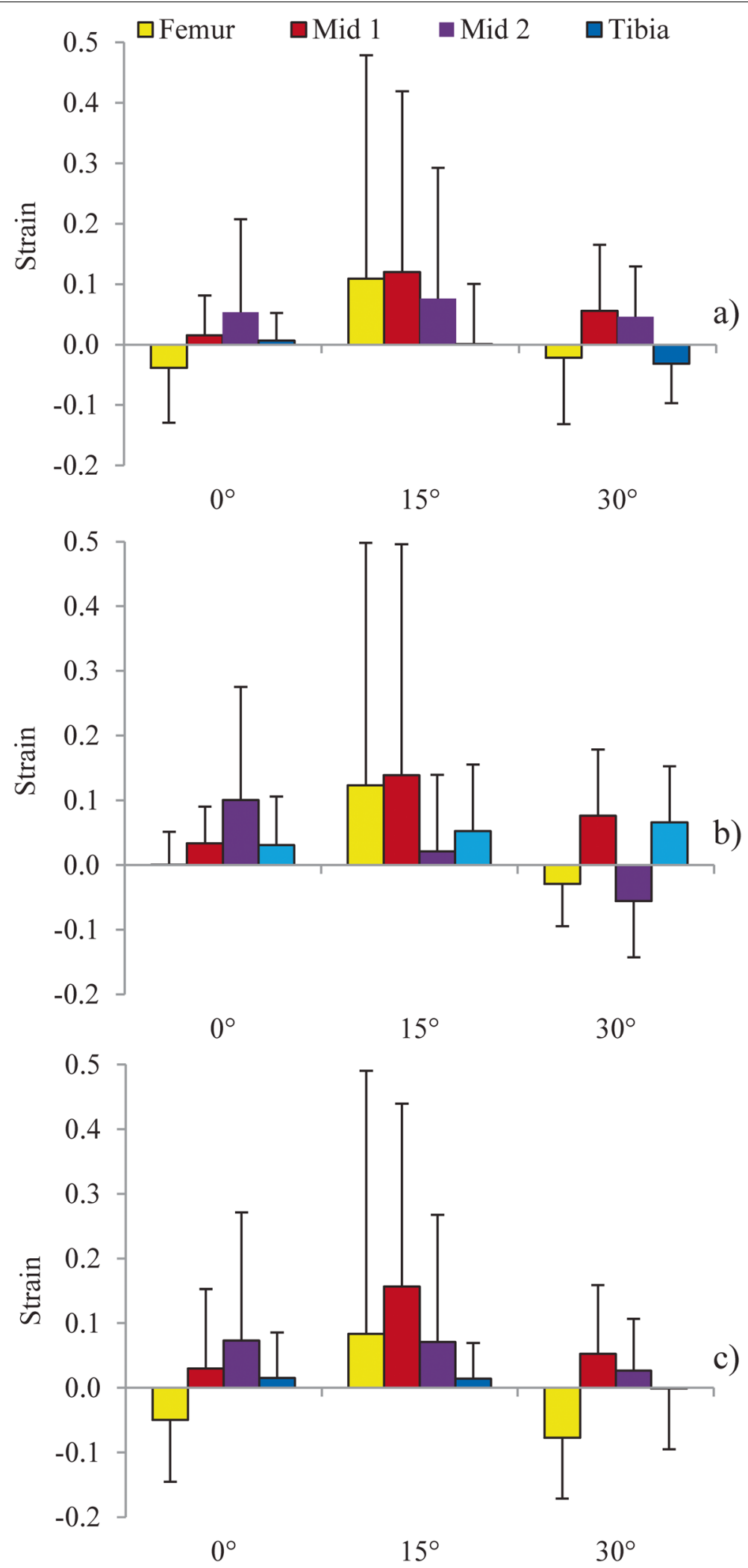

Fig. 4 Comparison of the mean (SD) magnitude of trans-axial strain between $A C L$ regions and across knee flexion angles when exposed to: $\mathbf{a} 5 \mathrm{Nm}$ internal rotation; b $100 \mathrm{~N}$ anterior translation; and $\mathbf{c}$ simulated pivot shift 
visualized the anterior surface of the ligament with an arthroscope and, while this was the optimal view to insert the majority of the markers, the femoral insertion was difficult to view from this angle. As a result, some markers were inserted without direct line of sight. Finally, the method, as presented here, is limited to investigating joint flexion angles from 0 to $30^{\circ}$ due to limited physical space within this specific micro-CT scanner. Despite this limitation, many physiologically important joint activities and a clinically important assessment (i.e., the pivot shift) occur in this flexion range. In addition, this dynamic joint motion simulator system is highly portable and is compatible with larger bore diameter CT-scanners.

\section{Conclusions}

Overall, the use of embedded radiopaque markers, micro-CT imaging, and a micro-CT compatible dynamic knee joint motion simulator provides a novel and highly reliable method for measuring regional ligament strain at clinically relevant loading conditions. The use of this minimally-invasive strain measurement technique at joint loads that are seen during regular joint use provides the opportunity to fully characterize intact ACL strain across various regions in the tissue at valid strain magnitudes, or to investigate the regional properties of novel and existing graft tissues designed to replace the ACL during reconstruction.

\section{Acknowledgements}

The authors would like to thank Steven Pollman for assistance with image reconstruction, and Mark Abbott for assistance with specimen collection.

\section{Authors' contributions}

Ms Blokker: Experimental design, specimen preparation, data collection and analysis, manuscript preparation. Dr. Wood: Specimen preparation, data collection, manuscript review. Mr. Milner: Design of bead detection and strain calculation software, manuscript review. Dr. Holdsworth: Conceptual design, experimental design, data analysis, manuscript review. Dr. Burkhart: Conceptual design, experimental design, data analysis, manuscript preparation and review. Dr. Getgood: Conceptual design, experimental design, manuscript preparation and review. The author(s) read and approved the final manuscript.

\section{Funding}

The authors would also like to acknowledge funding from the CIHR (Canadian Research Leaders in Musculoskeletal Health, PCS 150478, Dynamic Imaging of Musculoskeletal Systems, FDN148474), the Ontario Research Fund: Research Excellence (RE07-066), and the Institution's Bone and Joint Institute.

\section{Availability of data and materials}

The datasets used and/or analysed during the current study are available from the corresponding author on reasonable request.

\section{Declarations}

Ethics approval and consent to participate The cadaveric specimens were used in accordance with tissue use and ethical guidelines (Approval Number: MW 030217).

\section{Consent for publication}

N/A

\section{Competing interests}

Dr. Getgood and Dr. Burkhart received research funding for this study from Smith and Nephew and ConMed Linvatec and Dr. Burkhart also received salary support from Smith and Nephew. Dr. Getgood is a Consulting surgeon with Smith and Nephew and Ossur Inc.

\section{Author details}

${ }^{1}$ School of Biomedical Engineering, Western University, London, ON, Canada. ${ }^{2}$ Fowler Kennedy Sports Medicine Institute, Western University, London, ON, Canada. ${ }^{3}$ Robarts Research Institute, Western University, London, ON, Canada. ${ }^{4}$ Department of Medical Biophysics, Western University, London, ON, Canada. ${ }^{5}$ Kinesiology and Physical Education, University of Toronto, 55 Harbord St, Toronto, ON M5S 2W6, Canada. ${ }^{6}$ Fowler Kennedy Sport Medicine Clinic, London, ON, Canada. ${ }^{7}$ Department of Surgery, Western University, London, ON, Canada.

Received: 30 July 2021 Accepted: 12 October 2021

Published online: 22 October 2021

References

1. Anderson MJ, Browning WM, Urband CE, Kluczynski MA, Bisson LJ (2016) A systematic summary of systematic reviews on the topic of the anterior cruciate ligament. Orthop J Sport Med 4:1-23

2. Andriacchi TP, Briant PL, Bevill SL, Koo S (2006) Rotational changes at the knee after ACL injury cause cartilage thinning. Clin Orthop Relat Res:39-44. https://doi.org/10.1097/01.blo.0000197079.26600.09

3. Andriacchi TP, Mundermann A, Smith RL, Alexander EJ, Dyrby CO, Koo S (2004) A framework for the in vivo pathomechanics of osteoarthritis at the knee. Ann Biomed Eng 32:447-457

4. Beaulieu ML, Haladik JA, Bey MJ, McLean SG (2012) Validation of a novel method for quantifying and comparing regional $A C L$ elongations during uniaxial tensile loading. J Biomech 45:2710-2714. https://doi.org/10. 1016/j.jbiomech.2012.08.016

5. Berns GS, Hull ML, Patterson HA (1992) Strain in the anteromedial bundle of the anterior cruciate ligament under combined loading conditions. J Orthop Res 10:167-176. https://doi.org/10.1002/jor.1100100203

6. Beynnon DB, Fleming BC (1998) Anterior cruciate ligament strain in-Vivo : a review of previous work. J Biomech 31:519-525. https://doi.org/10. 1016/S0021-9290(98)00044-X

7. Blokker A, Getgood A, Curiale N, Nikolov H, Laing J, Holdsworth DW, Burkhart TA (2019) Development and assessment of a microcomputed tomogrpahy compatible five degrees-of-freedom knee joint motion simulator. J Biomech Eng 141:1-10

8. Blokker AM, Getgood A, Nguyen D, Holdsworth DA, Burkhart TA (2020) Insertion of small diameter radiopaque tracking beads into the anterior cruciate ligament results in repeatable strain measurement without affecting the material properties. Ann Biomed Eng. https://doi.org/10. 1007/s10439-020-02511-2

9. Blokker AM, Getgood AM, Nguyen D, Burkhart TA, Holdsworth DW (2021) Accuracy and precision of image-based strain measurement using embedded radiopaque markers. Med Eng Phys 92:88-92. https://doi.org/ 10.1016/j.medengphy.2021.05.005

10. Butler DL, Sheh MY, Stouffer DC, Samaranayake VA, Levy MS (1990) Surface strain variation in human patellar tendon and knee cruciate ligaments. J Biomech Eng 112:38-45

11. Fleiss JL, Levin B, Paik MC (2003) Statistical methods for rates and proportions, 3rd edn. Wiley, New Jersey

12. Hirokawa S, Yamamoto K, Kawada T (2001) Circumferential measurement and analysis of strain distribution in the human ACL using a photoelastic coating method. J Biomech 34:1135-1143. https://doi.org/10.1016/ S0021-9290(01)00078-1

13. Hosseini A, Lodhia P, Van De Velde SK, Asnis PD, Zarins B, Gill TJ, Li G (2012) Tunnel position and graft orientation in failed anterior cruciate ligament reconstruction: a clinical and imaging analysis. Int Orthop 36:845-852. https://doi.org/10.1007/s00264-011-1333-4

14. Khan R, Konyves A, Rama KRBS, Thomas R, Amis AA (2006) RSA can measure $A C L$ graft stretching and migration: development of a new method. Clin Orthop Relat Res:139-145. https://doi.org/10.1097/01.blo.00002 24016.42669.17 
15. Lalone EA, Peters TM, King GW, Johnson JA (2012) Accuracy assessment of an imaging technique to examine ulnohumeral joint congruency during elbow flexion. Comput Aided Surg 17:142-152. https://doi.org/10. 3109/10929088.2012.673638

16. Lebel BP, Pineau V, Gouzy SL, Geais L, Parienti JJM, Dutheil JJP, Vielpeau CH (2011) Total knee arthroplasty three-dimensional kinematic estimation prevision. From a two-dimensional fluoroscopy acquired dynamic model. Orthop Traumatol Surg Res 97:111-120. https://doi.org/10.1016/j.otsr. 2011.01 .003

17. Luque-Seron JA, Medina-Porqueres I (2016) Anterior cruciate ligament strain in vivo: a systematic review. Sports Health 8:451-455. https://doi. org/10.1177/1941738116658006

18. Maher JM, Markey JC, Ebert-May D (2013) The other half of the story: effect size analysis in quantitative research. CBE Life Sci Educ 12:345-351. https://doi.org/10.1187/cbe.13-04-0082

19. McLean SG, Mallett KF, Arruda EM (2015) Deconstructing the anterior cruciate ligament: what ee know and do not know about function, material properties, and injury mechanics. J Biomech Eng 137:020906. https://doi. org/10.1115/1.4029278

20. Samitier G, Marcano Al, Alentorn-Geli E, Cugat R, Farmer KW, Moser MW (2015) Failure of anterior cruciate ligament reconstruction. Arch Bone Jt Surg 3:220-240. https://doi.org/10.1016/j.csm.2012.08.015
21. Sharma L, Songn J, Felson DT, Cahue S, Shamiyeg E, Dunlop DD (2001) The role of knee alignment in disease progression and functional decline in knee osteoarthritis. JAMA 286:188. https://doi.org/10.1001/jama.286.2. 188

22. Stergiou N, Ristanis S, Moraiti C, Georgoulis AD (2007) Tibial rotation in anterior cruciate ligament (ACL)-deficient and $\mathrm{ACL}$-reconstructed knees: a theoretical proposition for the development of osteoarthritis. Sport Med 37:601-613. https://doi.org/10.2165/00007256-200737070-00004

23. Tashman S, Collon D, Anderson K, Kolowich P, Anderst W (2004) Abnormal rotational knee motion during running after anterior cruciate ligament reconstruction. Am J Sports Med 32:975-983. https://doi.org/10.1177/ 0363546503261709

\section{Publisher's Note}

Springer Nature remains neutral with regard to jurisdictional claims in published maps and institutional affiliations.

\section{Submit your manuscript to a SpringerOpen ${ }^{\circ}$ journal and benefit from:}

- Convenient online submission

- Rigorous peer review

- Open access: articles freely available online

- High visibility within the field

- Retaining the copyright to your article

Submit your next manuscript at $\boldsymbol{\nabla}$ springeropen.com 